\title{
AVALIAÇÃO DE UMA COLÔNIA DE AEDES AEGYPTI SELECIONADA CONTINUAMENTE COM O BIOLARVICIDA BACILLUS THURINGIENSIS SVAR. ISRAELENSIS
}

\author{
Vitória Maciel ${ }^{1, *}$, Karine Carvalho², Maria Helena Neves ${ }^{3}$ \\ ${ }^{1}$ Depto. de Entomologia, Centro de Pesquisas Aggeu Magalhães- FIOCRUZ, Recife-PE, Brasil \\ *vitoriamaciiel@hotmail.com
}

\section{INTRODUÇÃO}

O B. thuringiensis sorovariedade israelensis (Bti) é um importante agente de controle de larvas de Aedes aegypti. A sua atividade larvicida deve-se a cristais produzidos durante a esporulação que possuem quatro protoxinas denominadas Cry4A, Cry4B, Cry $11 \mathrm{~A}$ e Cyt1A (Fig. 1). O modo de ação do Bti consiste das seguintes etapas: ingestão dos cristais, processamento intestinal das protoxinas em toxinas e ligação a receptores específicos. O Bti tem sendo utilizado com sucesso desde 1980 sem registro de resistência e isso se deve, sobretudo, ao complexo modo de ação de suas mú Itiplas toxinas (Regis et al., 2000; Wirth, 2010). Apesar do baixo potencial de resistência associado ao uso do Bti, é necessário avaliar este risco devido à capacidade adaptativa das populações de Aedes, e pelo fato do tratamento com Bti ser contínuo em regiõ es tropicais que favorecem a proliferação do vetor durante todo o ano.
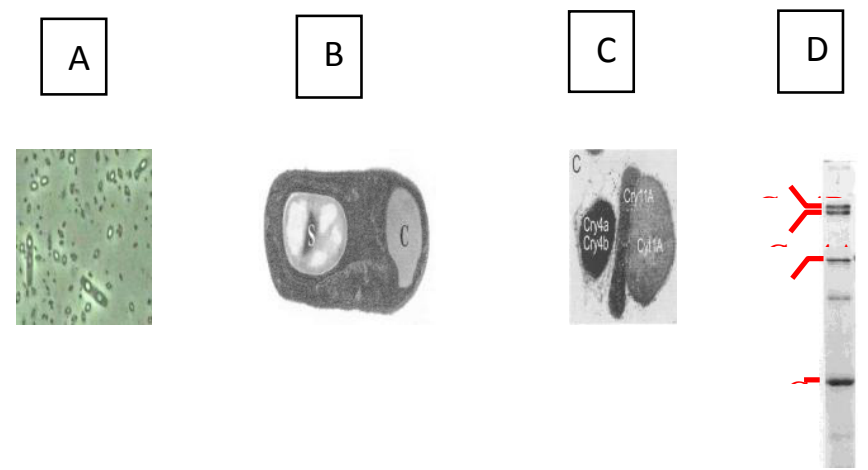

Fig.1. Bacillus thuringiensis israelensis. A. Cultura. B. Célula em esporulação com esporo (S) e cristal (C) C. Cristal e protoxinas. D. Perfil em SDS-PAGE das protoxinas do cristal.

Sendo assim, o principal objetivo do meu trabalho é avaliar a colô nia de Ae. aegypti (RecBti) exposta continuamente ao Bti em laboratório, simulando a pressão de seleção de uma rotina de tratamentos em campo. Os seguintes aspectos foram avaliados: suscetibilidade ao Bti, às toxinas isoladas do Bti, ao temephos e padrão de atividade de enzimas detoxificadoras de inseticidas.

\section{MATERIAIS E MÉTODOS}

Colônias. Rockefeller (referência) e RecBti (teste) foram mantidas sob condições de insetário.

Inseticidas. Bti Vectobac WDG ${ }^{\circledR}$ foi usado na seleção, e pós té cnicos de Bti (IPS82), de toxinas recombinantes Cry11A e Cry4B e de temephos (Sigma) nos bioensaios.

Seleção de larvas. A RecBti foi obtida a partir de ovos coletados em Recife. Amostras de no mínimo 5.000 larvas ( $3^{\circ}$ instar) de cada geração foram tratadas com uma dose de VectoBac ${ }^{\circledR}$ capaz de causar uma mortalidade $>50 \%$ após $24 \mathrm{~h}$. Os sobreviventes foram mantidos até a fase adulta para formar a geração seguinte (Fig. 2). Bioensaio. As concentrações letais para $50 \%\left(\mathrm{CL}_{50}\right)$ e $90 \%\left(\mathrm{CL}_{90}\right)$ de larvas expostas aos compostos testados foram através da análise de próbites.

Atividade de enzimas detoxificadoras. A atividade de glutationaS-transferases (GST), a-esterases, B-esterases, e oxidases de funçã o mista (MFO) foram avaliadas a partir de protocolo adaptado (Brasil, 2006), para larvas.

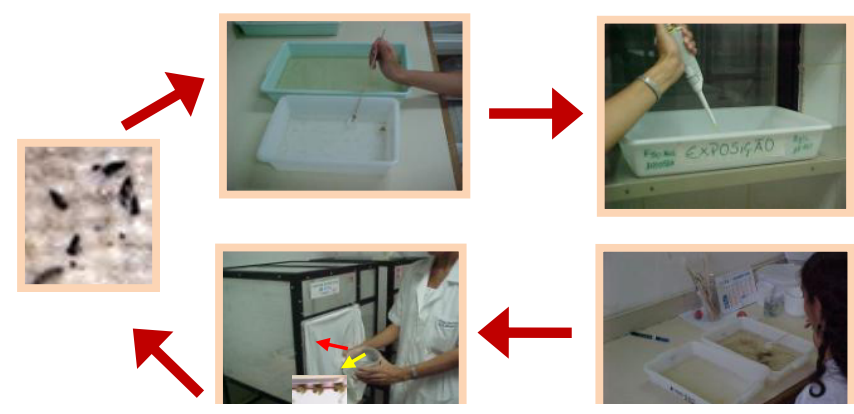

Fig.2. Principais etapas do procedimento de seleção de larvas colônia RecBti com Bti (Vetobac®).

\section{RESULTADOS E DISCUSSÃO}

Seleção da colônia RecBti com Bti

A colônia RecBti foi selecionada, até o momento, por 26 gerações. Em cada geração pelo menos 5.000 larvas do $3^{\circ}$ estádio foram tratadas (Bti-VectoBac WG $®$ ), totalizando mais de 260.000 larvas expostas e a mortalidade média por geração foi de $75 \%$.

Suscetibilidade de RecBti ao Bti, às toxinas Cry11Aa, Cry4Aa e temephos

(c) 2018 CC BY-NC-ND LICENSE ANAIS: ENCONTRO ANUAL DA BIOFÍSICA (2018): 101-102 BIOFÍ́ICA E RADIOBIOLOGIA, BIOCIÊNCIAS, UFPE 05 E 06 DE FEVEREIRO, RECIFE, PERNAMBUCO, BRASIL ISSN: 2526-6071 
A suscetibilidade das larvas RecBti ao Bti e à toxina Cry4Ba não apresentou alterações significativas, pois, as razões de toxicidade ( $\mathrm{RT}=\mathrm{CL}$ colônia teste/CL colônia referência) mostraram aumentos inferiores a cinco vezes (Fig. 3) nas gerações analisadas. A suscetibilidade ao temephos, analisada na $F_{19}$ e $F_{25}$ foi similar à colônia de referência e a razão RT entre as concentrações letais foi inferior a 2 (Tabela 1).

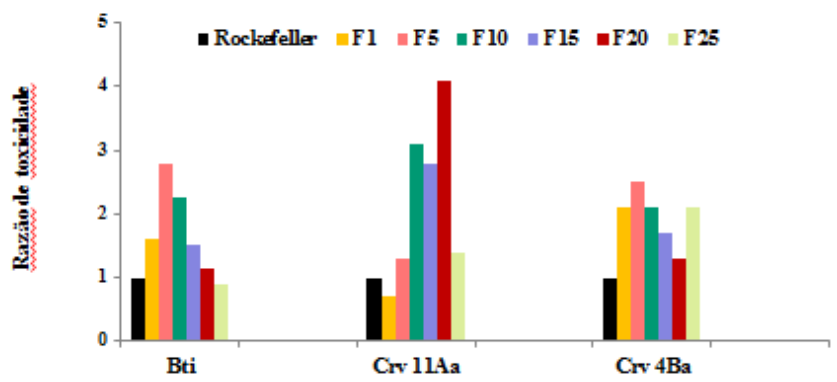

Fig. 3. Razão de toxicidade entre as $L C_{50}$ do Bti, Cry11Aa e Cry4Ba, para larvas da colônia RecBti, e as respectivas $\mathrm{LC}_{50}$ para larvas da colônia Rockefeller

Tabela 1. Toxicidade do temephos para larvas do $3^{\circ}$ instar de Aedes aegypti da colô nia RecBti $\left(F_{19}\right.$ e $\left.F_{25}\right)$ em relação à Rockefeller. $R T=C L$ RecBti/CL Rockefeller.

\begin{tabular}{lllll}
\hline Colônia & CL $_{50}$ (IC 95\%) & RT $^{b}$ & CL $_{90}$ (IC 95\%) & RT \\
Rockefeller & $0,007(0,007-0,008)$ & 1,0 & $0,010(0,010-0,011)$ & 1,0 \\
RecBti F & $0,007(0,007-0,008)$ & 1,0 & $0,016(0,012-0,018)$ & 1,6 \\
RecBti F F & $0,008(0,007-0,008)$ & 1,0 & $0,014(0,012-0,016)$ & 1,3 \\
\hline
\end{tabular}

\section{Atividade de enzimas detoxificadoras}

Os dados mostram que larvas RecBti da $F_{15}$ não possuem alterações na atividade das a-esterases e MFO porém apresentaram aumento de $B$-esterases e GSTs na $F_{15}$ e de $B$-esterases na $F_{25}$, em relação à referência Rockefeller. As alterações não estão correlacionadas com a suscetibilidade de larvas Rec-Bti ao temephos, que foi similar.

Tabela 2. Atividade de enzimas detoxificadoras em larvas de Aedes aegypti da colônia Rec_Bti nas $F_{15}$ e $F_{25}$. Classificação $(\mathrm{Cl})$ em relação à colônia Rockefeller : $A=$ alterada, I= inalterada.

\begin{tabular}{|c|c|c|c|c|c|c|c|c|c|c|c|c|}
\hline \multicolumn{2}{|c|}{ Enzimas } & \multicolumn{2}{|c|}{ GST } & \multirow[b]{2}{*}{$\mathrm{N}^{\circ}$} & \multicolumn{2}{|c|}{ a-ester } & \multirow[b]{2}{*}{$\mathbf{N}^{\circ}$} & \multicolumn{2}{|c|}{$\beta$-ester } & \multirow[b]{2}{*}{$\mathbf{N}^{\circ}$} & \multicolumn{2}{|c|}{ MFO } \\
\hline $\mathrm{Ge}$ & $\mathbf{N}^{\circ}$ & $\Rightarrow 99$ & $\mathrm{Cl}$ & & $\Rightarrow 99$ & $\mathrm{Cl}$ & & $\begin{array}{c}\Rightarrow 9 \\
9\end{array}$ & $\mathrm{Cl}$ & & $\Rightarrow 99$ & $\mathrm{Cl}$ \\
\hline$F_{15}$ & 160 & 28 & A & 209 & 3 & I & 122 & 37 & A & 126 & 5 & 1 \\
\hline$F_{25}$ & 113 & 1 & I & 160 & 0 & I & 159 & 27 & A & 82 & 0 & I \\
\hline
\end{tabular}

\section{CONCLUSÕES}

Larvas RecBti expostas ao Bti por 26 gerações contínuas não apresentam alterações de suscetibilidade a este agente ou às suas toxinas.

As larvas são suscetíveis ao temephos, apesar do aumento da atividade de GSTs e B-esterases, não tendo sido observada uma correlação entre estes parâmetros.

\section{REFERÊNCIAS}

Amorim, L.B., Oliveira, C.M.F., Rios, E.M., Regis, L., Silitha, M.H.N.L., 2007. Development of Culex quinquefasciatus resistance to Bacillus sphaericus strain IAB59 needs long term selection pressure. Biological Control 42, 155-160.

Brasil, 2006. Metodologia para quantificação de atividade de enzimas relacionadas com a resistência a inseticidas em Aedes aegypti. Secretaria de Vigilância em Sáude, Ministério da Saúde, Brasília (Brazil).

Brogdon, W.G., McAllister, J.C., 1998. Insecticide resistance and vector control. Emerg. Infect. Dis. 4, 605-613.

Burt, F.J., Rolph, M.S., Rulli, N.E., Mahalingam, S., Heise, M.T., 2012. Chikungunya: a re-emerging virus. Lancet 379, 662-671.

Crickmore, N., Bone, E.J., Wiliams, J.A., Ellar, D.J., 1995. Contribution of the individual components of the delta-endotoxin crystal to the mosquitocidal activity of Bacillus thuringiensis subs. israelensis. FEMS Microbiol Lett 131, 249-254.

de Barros Moreira Beltrão, H., Silva-Filha, M.H., 2007. Interaction of Bacillus thuringiensis svar. israelensis Cry toxins with binding sites from Aedes aegypti (Diptera: Culicidae) larvae midgut. FEMS microbiology letters 266, 163-169.

Guidi, V., Patocchi, N., Luthy, P., Tonolla, M., 2011. Distribution of Bacillus thuringiensis subsp. israelensis in soil of a swiss wetland reserve after 22 years of mosquito control. Appl. Environ. Microbiol. 77, 3663-3668.

Hertlein, M.B., Mavrotas, C., Jousseaume, C., Lysandrou, M., Thompson, G.D., Jany, W., Ritchie, S.A., 2010. A review of spinosad as a natural product for larval mosquito control. J. Am. Mosq Control. Assoc. 26, 67-87.

Lacey, L.A., 2007. Bacillus thuringiensis serovariety israelensis and Bacillus sphaericus for mosquito control. J. Am. Mosq. Control Assoc. 23, 133-163.

Regis, L., da Silva, S.B., Melo-Santos, M.A., 2000. The use of bacterial larvicides in mosquito and black fly control programmes in Brazil. Mem. Inst. Oswaldo Cruz 95 Suppl 1, 207-210.

Soberón, M., Fernández, L.E., Pérez, C., Gill, S.S., Bravo, A., 2007. Mode of action of mosquitocidal Bacillus thuringiensis toxins. Toxicon 49, 597-600.

Teixeira, M.G., Barreto, M.L., 2009. Diagnosis and management of dengue. BMJ 339, b4338.

WHO, 1985. Informal consultation on the development of Bacillus sphaericus as microbial larvicide, World Health Organization, pp. TDR/BCV/SPHAERICUS/85.83.81-24.

Wirth, M.C., 2010. Mosquito resistance to bacterial larvicidal proteins. Open J Toxicol 3, 101-115.

Wirth, M.C., Walton, W.E., Federici, B.A., 2010. Inheritance patterns, dominance, stability, and allelism of insecticide resistance and cross-resistance in two colonies of Culex quinquefasciatus (Diptera: Culicidae) selected with cry toxins from Bacillus thuringiensis subsp, israelensis. J Med Entomol 47, 814822. 